Proc. Indian Acad. Sci. (Earth Planet. Sci.) Vol, 91, No. 1, March 1982, pp. 29-41. (c) Printed in India.

\title{
Fast automatic solution of the inverse resistivity problem
}

\author{
SRI NIWAS, PAWAN KUMAR and H R WASON \\ Department of Earth Sciences, University of Roorkee, Roorkee 247 672, India
}

MS received 27 March 1981; revised 8 September 1981

\begin{abstract}
The paper presents a fast automatic approach to solve the inverse resistivity problem, assisted by optimization, which is a non-linear model-fitting technique. The selected inverse problems are ill-posed and the inverse solution is defined by 'best fit' in the sense of least-squares. Formulations are presented in a systematic manner for Newton's method, least squares method and Marquardt's modification (ridge regression) method based on local linearization of non-linear problem. The convergence of least-squares method and Marquardt's method, to provide a robust solution, are first tested on a theoretical model and effectiveness of Marquardt's method is demonstrated, and then two-field apparent resistivity curves from Banda district, India are interpreted and discussed.
\end{abstract}

Keywords. Resistivity; inverse solution.

\section{Introduction}

The conventional indirect methods of interpretation of resistivity sounding data such as curve matching have very low resolving power. The more powerful direct method of interpretation as suggested by Slichter (1933) is not easy to handle, however, because of the "ill-posed "nature of the inverse problems and the associated computational difficulties.

The direct interpretation consists of two steps--viz:

(i) extraction of Kernel function $K(\lambda)$ from the field measurement and (ii) decomposition of Kernel function in terms of layer parameters. With the work of Ghosh (1971), step (i) is perfected quite satisfactorily. However, the most important step (ii) could not be improved earlier due to lack of computational facilities. Recently, a number of workers have taken up the job of automatic interpretation of resistivity data (Zohdy 1974; Bichara and Lakshmanan 1976; Inman et al 1973; Johanson. 1977). Keofoed (1979) has given a brief account of all the work pertaining to direct interpretation. However, only Bichara and Lakshmanan (1976) have used the resistivity transform function $T(1 / \lambda)$ (a function related with Kernel function; Koefoed, 1970) as an intermediary step. The essential advantage of using the resistivity transform function as an intermediary step lies in the fact that it has dimensions of resistivity and is solely determined by the layer distribution and is independent of electrode configuration. However, the resistivity transform function shows the same asymptotic behaviour as the apparent resistivity function, both for small and for large abscisa values. 
The resistivity transform function can be extracted from the apparent resistivity function by the application of a simple linear filter using Ghosh (1971) formula

$$
T_{m}=\sum_{j=-\infty}^{\infty} a_{j} R_{m-j},
$$

where,

$T_{m}$ is the resistivity transform at sample point $m$,

$m$ is the number of sample points,

$a_{j}$ is the filter coefficients, number determined by the length of the filter to be used, and

$R_{m}$ is the apparent resistivity value at sample point $m$.

In the present paper, an initial earth model is assumed. The resistivity transform function of this model is calculated and compared with the one calculated from the field sounding curve. The differences of the transforms are used to automatically modify the parameters of the earth model, with the use of Marquardt's algorithm.

\section{Formulation of the problem}

\subsection{Inverse problem}

The purpose of solving the resistivity inverse problem of a horizontally layered earth is to obtain a model with layer parameters $d_{1}, d_{2}, \ldots, d_{n-1}$ (layer thicknesses) and $\rho_{1}, \rho_{2}, \ldots, \rho_{n}$ (layer resistivities) so that the computed resistivity transform function $T_{c}\left(u, d_{1}, \ldots, d_{n-1}, \rho_{1}, \ldots, \rho_{n}\right)$ for this model and the observed resistivity transform function $T_{0}(u)$ will coincide for all values of $u \in u_{f}$. Here $u=1 / \lambda$ and $u_{f}$ denotes the set of all $u$ for which the observed resistivity transform can be determined. Thus, for the sought for model, the following equality is required.

$$
T_{c}\left(u, d_{1}, d_{2}, \ldots, d_{n-1}, \rho_{1}, \rho_{2}, \ldots, \rho_{n}\right)=T_{0}(u), u \in u_{f}
$$

However, to achieve the equality (2) is rather not possible as resistivity inverse problem is a non-linear problem. Thus the methods based on the local linearization of (2) can be adopted to exploit the well developed theories of linear operators on Hilbert space. If the observed values of the resistivity transform $T_{0}(u)$ are determined in a discrete set of $u_{f}=\left\{u_{1}, u_{2}, u_{3}, \ldots, u_{m}\right\}$, then (2) represents a system of $m$ nonlinear equations in $2 n-1$ unknown layer parameters $d_{1}, d_{2}, \ldots, d_{n-1}, \rho_{1}, \rho_{2}, \ldots, \rho_{n}$. Let $d_{1}^{(0)}, d_{2}^{(0)}, \ldots, d_{n-1}^{(0)}, \rho_{1}^{(0)}, \rho_{2}^{(0)}, \ldots \rho_{n}^{(0)}$, be an estimated solution of layer parameters close enough to the parameters of the true geoelectric structure $d_{1}, d_{2}, \ldots$, $d_{n-1}, \rho_{1}, \rho_{2}, \ldots, \rho_{n}$. Equation (2) can be linearized by expanding it in Taylor series and retaining only the first derivative term. 


$$
\begin{aligned}
& T_{0}\left(u_{1}\right)=T_{c}\left(u_{i}, d_{1}^{(0)}, d_{2}^{(0)}, \ldots, d_{n-1}^{(0)}, \rho_{1}^{(0)}, \rho_{2}^{(0)}, \ldots, \rho_{n}^{(0)}\right) \\
& +\sum_{j=1}^{n-1}\left(d_{j}-d_{j}^{(0)}\right)\left[\partial T _ { c } \left(u_{i}, d_{1}^{(0)}, d_{2}^{(0)}, \ldots, d_{n-1}^{(0)}, \rho_{1}^{(0)}, \rho_{2}^{(0)},\right.\right. \\
& \left.\left.\quad \ldots \rho_{n}^{(0)}\right) \mid \partial d_{j}^{(0)}\right]+\sum_{j=1}^{n}\left(\rho_{j}-\rho_{j}^{(0)}\right)\left[\partial T _ { c } \left(u_{i}, d_{1}^{(0)} d_{2}^{(0)}, \ldots,\right.\right. \\
& \left.\left.\quad d_{n-1}^{(0)}, \rho_{1}^{(0)}, \ldots, \rho_{n}^{(0)}\right) / \partial \rho_{j}\right], i=1,2, \ldots m .
\end{aligned}
$$

Introducing the $m$ data values $T_{0}\left(u_{1}\right), T_{0}\left(u_{2}\right) \ldots T_{0}\left(u_{m}\right)$ corresponding to $m$ sample points as the vector $\mathbf{T}_{0}(u)=\left[T_{0}\left(u_{1}\right), T_{0}\left(u_{2}\right), \ldots, T_{0}\left(u_{m}\right)\right]^{T}$ and the restricted earth model, determined by $2 n-1$ free parameters as the vector $\mathbf{P}=\left[d_{1}, d_{2}, \ldots, d_{n-1}\right.$, $\left.\rho_{1} \rho_{2}, \ldots \ldots \rho_{n}\right]^{T}$, we assume that the forward problem generates a set of model data for each setting $\mathbf{P}$ which can be denoted as a vector function by $\mathbf{T}_{c}(P)=\left[T_{c}\left(u_{1}, P\right)\right.$, $T_{c}\left(u_{2}, P\right), \ldots,\left.T_{c}\left(u_{m}, P\right)\right|^{T}$, where $T$ indicates the operation of matrix transposition.

Now we can define an influence matrix $A[\mathbf{P}]$ with the elements

$$
\left[a_{i j}\right]=\left\{\begin{array}{l}
\frac{\partial T_{c}\left(u_{1}, \mathbf{P}^{(0)}\right)}{\partial d_{j}}, j=1, \ldots, n-1 i=1,2, \ldots . m, \\
\frac{\partial T_{c}\left(u_{i}, \mathbf{P}^{(0)}\right)}{\partial d_{j \sim n}}, j=n+1, n+2, \ldots, 2 n .
\end{array}\right.
$$

Using this notion, if $m=2 n-1$ we can then define an iteration process according to (3),

$$
\mathbf{P}=\mathbf{P}^{(0)}+\left\{A\left[\mathbf{P}^{(0)}\right]\right\}^{-1}\left\{T_{0}-T_{c}\left[\mathbf{P}^{(0)}\right]\right\},
$$

where $\mathbf{P}^{(0)}$ is the initial estimation of the parameters of the geoelectric section under investigation. The iterative method successively improves the old model to new model until the error measure is small and the parameters are stable with respect to reasonable changes in the model. Expression (5) represents the well-known Newton's method of solving inverse problems. The convergence of this iterative scheme very much depends on the computed resistivity transform, on the choice of the set $u_{f}$ (here $u_{f}$ is restricted by $m=2 n-1$ ). However, in this process the condition (2) is diffcult to achieve, and thus the iterative process is terminated as soon as,

$$
T_{\mathrm{D}}=\left\|T_{\mathrm{a}}-T_{c}\left[\mathbf{P}^{(0)}\right]\right\| 2 \leqslant \epsilon,
$$

where $\epsilon$ is the measure of the chosen accuracy. Application of Newton's method for solving field cases are somewhat restricted in the sense that all the observed data sets are not utilized (due to the condition $m=2 n-1$ ) and the method is sensitive to the choice of initial estimation (Pek and Červ 1979). 
The condition $m=2 n-1$ can be modified to $m>2 n-1$ by applying the leastsquares method. Thus entire data set can be accommodated in the scheme. The iteration process may be written in a modified form as

$$
\mathbf{P}=\mathbf{P}^{\{0)}+\left\{A^{T}\left[\mathbf{P}^{(0)}\right] A\left[\mathbf{P}^{(0)}\right]\right\}^{-1} A^{T}\left[\mathbf{P}^{(0)}\right]\left\{T_{0}-T_{c}\left[\mathbf{P}^{(0)}\right]\right\} .
$$

The factual meaning of expression (7) simply lies in the sense that the system of equations for the components of correction vector

$$
\Delta \mathrm{P}=\mathbf{P}-\mathbf{P}^{(0)},
$$

should be solved at every iteration step of

$$
\Delta \mathbf{P}=\left\{A^{T}\left[\mathrm{P}^{(0)}\right] A\left[\mathrm{P}^{(0)}\right]\right\}^{-1} A^{T}\left[\mathrm{P}^{(0)}\right]\left\{T_{0}-T_{c}\left[\mathbf{P}^{(0)}\right]\right\} .
$$

The system of equation (9) is obtained by setting the partial derivitive of the objective function

$$
T_{\mathrm{D}} \leqslant \sum_{i=1}^{m}\left(T_{0_{i}}-T_{c_{i}}\left[\mathrm{P}^{(0)}\right]\right)^{2}
$$

with respect to each unknown parameters to zero as

$$
\frac{\partial T_{D}}{\partial P_{j}}=0, \quad j=1,2, \ldots, 2 n-1
$$

where $n$ is the number of layers in the geoelectric section. In (9), $\left\{A^{T}\left[\mathbf{P}^{(0)}\right] A\left[\mathbf{P}^{(0)}\right]\right\}^{-1}$ $A^{T}\left[\mathrm{P}^{(0)}\right]$ is known as 'least-square estimator', and $A^{T}\left[\mathrm{P}\left({ }^{(0)}\right)\right]$ is the transpose of the matrix $A\left[\mathrm{P}^{(0)}\right]$.

Under this procedure we obtain the correction vector $\Delta \mathbf{P}$ to minimise $\Delta T\left(=T_{0}\right.$ $\left.-T_{c}\left[\mathrm{P}^{(0)}\right]\right)$ in the sense that

$$
\Delta T^{T} \Delta T \leqslant \epsilon .
$$

If convergence is achieved after a few iterations, the final unknown parameters can be obtained by the expression,

$$
\mathbf{P}_{j}=\mathbf{P}_{j}^{(0)}+\Delta \mathbf{P}_{j}, \quad j=1,2, \ldots, 2 n-1 .
$$

Although the region of convergence of this method is generally greater than that of Newton's method if the initial estimation of the solution is chosen in a close vicinity of the true geoelectric section. However, if the initial estimation is not very close to the true solution the obtained solution may attain a local minimum and thrown away from the actual minimum and hence from the true solution. 
Marquardt (1963) presented an algorithm to improve the convergence properties of the iteration scheme (9) to estimate non-linear parameters. Accordingly the following system of equations

$$
\left\{A^{T}\left[\mathbf{P}^{(0)}\right] A\left[\mathbf{P}^{(0)}\right]+\text { TLAM. } I\right\}^{-1} \Delta P=A^{T}\left[\mathbf{P}^{(0)}\right]\left\{T_{0}-T_{c}\left[\mathbf{P}^{(0)}\right]\right\},
$$

is used for the components of correction vector $\Delta \mathrm{P}$ to be solved at every iteration. Equation (13) can be rewritten as

$$
\Delta \mathrm{P}=\left\{A^{T}\left[\mathrm{P}^{(0)}\right] A\left[\mathbf{P}^{(0)}\right]+\text { TLAM } . I\right\}^{-1} A^{T}\left[\mathrm{P}^{(0)}\right]\left\{T_{0}-T_{\varepsilon}\left[\mathbf{P}^{(0)}\right]\right\}
$$

Here $I$ is the $(2 n-1) \times(2 n-1)$ identity matrix and TLAM is a fixed value known as Marquardt's Lamda. In expression (14) the term $\left\{A^{T}\left[\mathbf{P}^{(0)}\right] A\left[\mathrm{P}^{(0)}\right]+\text { TLAM.I }\right\}^{-1}$ $A^{T}\left[\mathrm{P}^{(0)}\right]$ is known as 'ridge regression estimator'.

The steepest descend method guarantees the convergence to the true solution if the initial estimation is within a reasonable range of true solution but the convergence is extremely slow after the first few iterations. The least squares method yields rapid rate of convergence but it requires the initial estimation to be very close to the true solution. Marquardt's method combines the advantages of the two methods provided the value of TLAM is properly chosen at every iteration. If TLAM $\rightarrow 0$, Marquardt's method reduces to the Newton's least squares method and if TLAM $\rightarrow \infty$ it works as the method of steepest descend, the optimum value of TLAM being 1.0 and 0.01 . However, Johanson (1977) modified this to permit an adaptive choice for the value of TLAM in successive iteration.

\subsection{Forward Problem}

The expression for resistivity transform function for the $n$-layer geoelectric section can be written following Koefoed (1970) which is utilized to compute $T_{c}\left[\mathbf{P}^{(0)}\right]$, as,

$$
T_{c(N)}\left[\mathbb{P}^{\left(0^{\prime}\right]}\right]=\frac{T^{\prime}(N)+T(N-1)}{1+\frac{T^{\prime}(N) T(N-1)}{\rho_{N}^{2}}}, \quad N=2, \ldots n,
$$

where $T^{\prime}(N)=\rho_{N} \tanh \left(\frac{d_{N-1}}{u_{f}}\right)$ and $T(N-1)$ is the resistivity transform of the same $n$-layer section whose top layer is removed. When $N=1$, resistivity transform takes the numerical value of resistivity of half-space. For successful use of (15) we start building the layered model from bottom to top i.e. the bottom layer is considered as first layer and top layer as the $n$th layer.

To construct influence matrix $A\left[\mathrm{P}^{(0)}\right]$, we need the partial derivative of resistivity transform function with respect to unknown parameters. Let us choose

$$
Q \in\left(\rho_{N^{\prime}} d_{N-1}\right)
$$

Proc.-3 
and $R \in\left(\rho_{1}, p_{2}, \ldots, \rho_{N-1}, d_{1}, d_{2}, \ldots, d_{N-2}\right)$, then from (15) we have the partial derivative as

$$
\frac{\partial T_{c}(N)}{\partial R}\left[u_{i}, \mathbf{P}^{(0)}\right]=\frac{\partial T(N-1)}{\partial R} \frac{1-T^{\prime 2}(N) / \rho_{N}^{a}}{\left(1+\frac{T^{\prime}(N)}{\rho_{N}^{2}} \cdot T(N-1)\right)^{2}}
$$

and

$$
\begin{aligned}
& \frac{\partial T_{c}(N)}{\partial Q}\left[u_{1} \mathbf{P}^{(0)}\right]=\frac{\frac{\partial T^{\prime}(N)}{\partial Q}}{\left[\left(1+\frac{T^{\prime}(N)}{\rho_{N}^{2}} \cdot T(N-1)\right)^{2}\right]} \\
& -\frac{\left(T^{\prime \prime}(N)+T(N-1)\right) T(N-1) \frac{\partial}{\partial Q}\left(T^{\prime}(N) / \rho_{N}^{2}\right)}{\left(1+\frac{T^{\prime}(N)}{\rho_{N}^{2}} \cdot T(N-1)\right)^{2}}
\end{aligned}
$$

\section{Nomerical results and discussion}

On the basis of developed expression in $\$ \S 2 \cdot 1$ and $2 \cdot 2$ a composite computer pro. grame is developed to determine observed resistivity transform from the apparent resistivity curve and subsequently to identify the resistivity transform function in unknown layer parameters of geoelectric section. The developed algorithm is first tested on theoretical resistivity sounding curve for the presumed model. Then two field sounding from Banda district are interpreted.

\subsection{Theoretical sounding curve}

The resistivity sounding curve is obtained for Schlumberger configuration over the presumed three layer model for half current electrode separation $(A B / 2)$ up to $1000 \mathrm{~m}$. The resistivities of the first $\left(\rho_{1}\right)$, second $\left(\rho_{2}\right)$ and third $\left(\rho_{3}\right)$ layer are taken to be equal to $100 \Omega \mathrm{m}, 4 \Omega \mathrm{m}$ and $20 \Omega \mathrm{m}$, whereas the thickness of first layer $\left(d_{1}\right)$ and second layer $\left(d_{2}\right)$ are taken to be $10 \mathrm{~m}$ and $30 \mathrm{~m}$ respectively. The obtained resistivity curve is given in figure 1. The resistivity transform functions are then extracted from the apparent resistivity curve using the Ghosh (1971) 9-point filter and sampled values of apparent resistivity with spacing $1 / 3$ ln (10). Here the formula used can be written from (1) as

$$
T_{m}=\sum_{j=-2}^{6} a_{j} R_{m-j}
$$

The extracted resistivity transform are also plotted $v s . u(=1 / \lambda)$ in figure 1 .

To compare, this model is interpreted using both Marquardt's method and least squares method. The starting model for both the methods is taken as $\rho_{l}=100 \Omega \mathrm{m}$, 


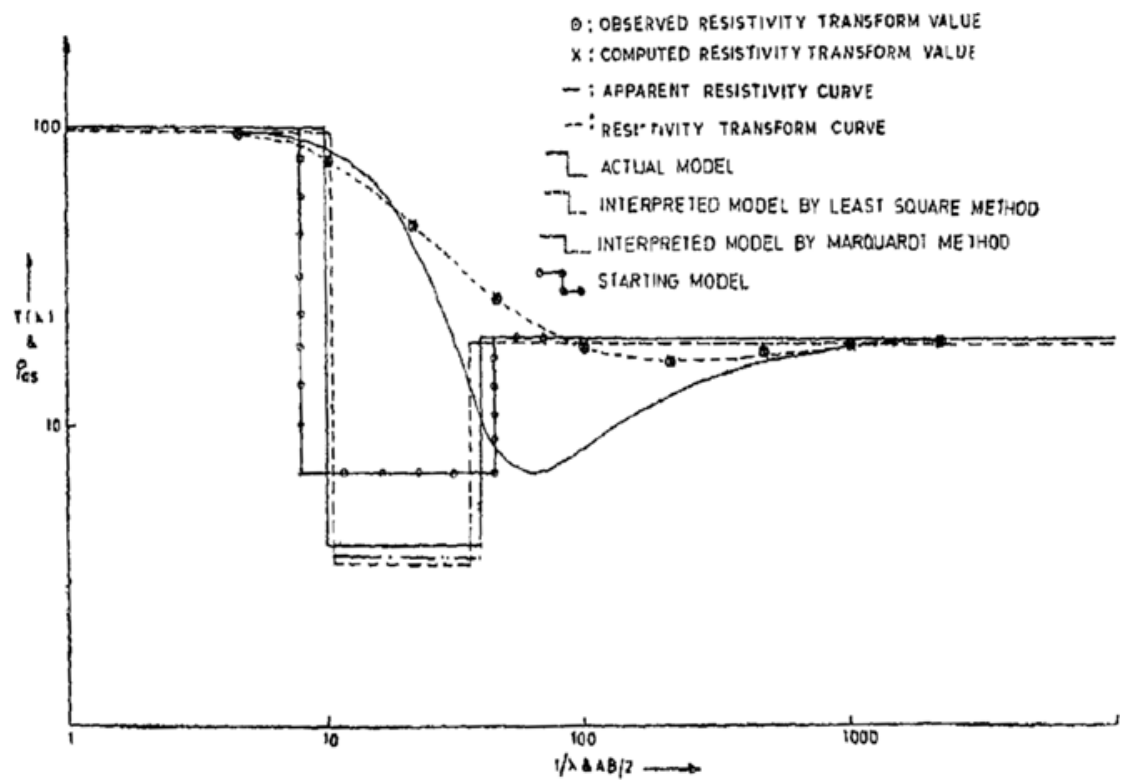

Figure 1. Plot of apparent resistivity curve, observed resistivity transform curve, computed resistivity transform curve, starting model and interpreted model using least squares method and Marquardt's method.

$p_{2}=7 \Omega \mathrm{m}, p_{3}=20 \Omega \mathrm{m}, d_{1}=8 \mathrm{~m}$ and $d_{2}=35 \mathrm{~m}$. These values are chosen by visual observation of apparent resistivity curve and by noting the point of inflexion. The estimation of starting model is certainly not difficult for experienced geophysicists. The initial value of TLAM is taken to be unity and four different rate of variation of TLAM in each advancing iterations viz. TLAM/1.2, TLAM/1.5, TLAM $/ 3$ and TLAM $/ 4$ are tested. The comparison of inverse solutions obtained using these four rates of variation show that TLAM/1.5 and TLAM/3 variation rates can be accepted. However, the variation rate TLAM/1.5 gives marginally better results than the TLAM/ 3 but the time taken is considerably more. So, for the present case the variation rate TLAM $/ 3$ is used.

In the present scheme of obtaining inverse solution, the initially estimated layer parameters of the geoelectric section are updated in each iteration and the $T_{D}$ values are minimised in the least square sense as

$$
\Delta T^{T} \Delta T=\sum_{i}\left(T_{c}-T_{c}\left[P^{(0)}\right]\right)^{2}=T_{D} \leqslant \epsilon
$$

The graphical plot of $T_{D}$ values vs. iteration number (figure 2) shows that the $T_{D}$ values in ridge regression method fall very rapidly from about 100 to a minimum $(0.022)$ in 5 iterations. After that the $T_{D}$ values start increasing to the highest value of 0.117 at 9 th iteration, after which they again starts decreasing to a value of 0.0205 at 12 th iteration. Beyond this the decrease rate is extremely slow as it reaches to a stable value of 0.0191 at 15 th iteration and afterward. The variation of $T_{D}$ with respect to iteration number for least squares method is also presented in figure 2 . 

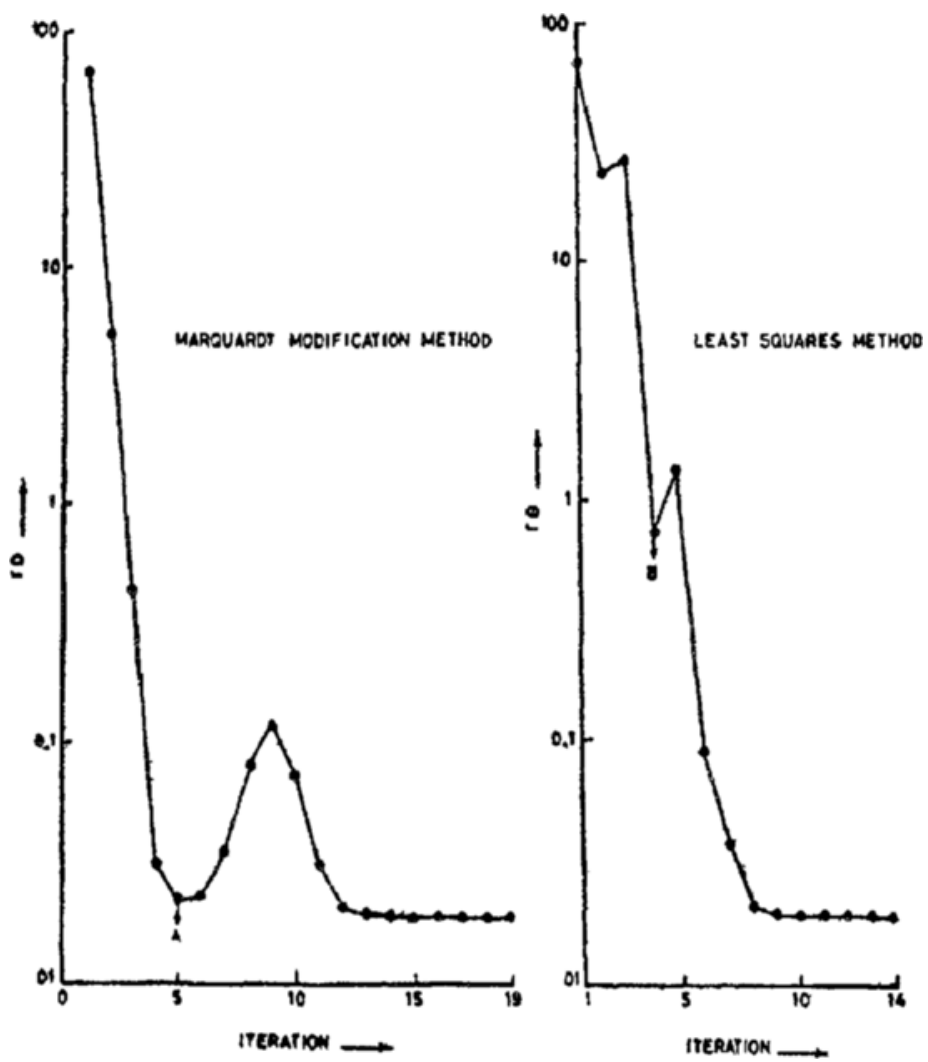

Figure 2. Plot of $T_{D}$ vs. iteration number for curvo in figur 1 for Marquardt's method and least squares method.

Figure 3 represents the comparative plot of the convergence of the layer parameters $\rho_{1}, \rho_{2}, \rho_{2}, d_{1}$ and $d_{2}$ for least squares method and ridge regression method. One can observe from figure 3 the smooth and perfect convergence of all the parameters in case of Marquardt's method in comparison to the corresponding parameters in least squares method. One can observe from this figure, from table 1 and from figure 2 that convergence is better in Marquardt's method in comparison to least squares method. It is also evident from figure 3 that when parameter $d_{2}$ starts converging, the parameter $\rho_{2}$ starts diverging and finally attains flat characteristics. The convergence line of $d_{2}$ and $\rho_{2}$ are almost parallel to each other. This might be due to a slight influence of equivalence as the resistivity of the intermediate layer is extremely low. The observed resistivity transform, computed resistivity transform, starting model and final inverse solutions are plotted in figure 1.

\subsection{Field sounding curve}

After gaining confidence on the theoretical sounding curve, we would like to demonstrate the application of Marquardt's method to obtain the solution of two field sounding curve. For inversion, two feld resistivity sounding curve from the Pahari Block of Banda district, India are considered. The area for geoelectric sounding 


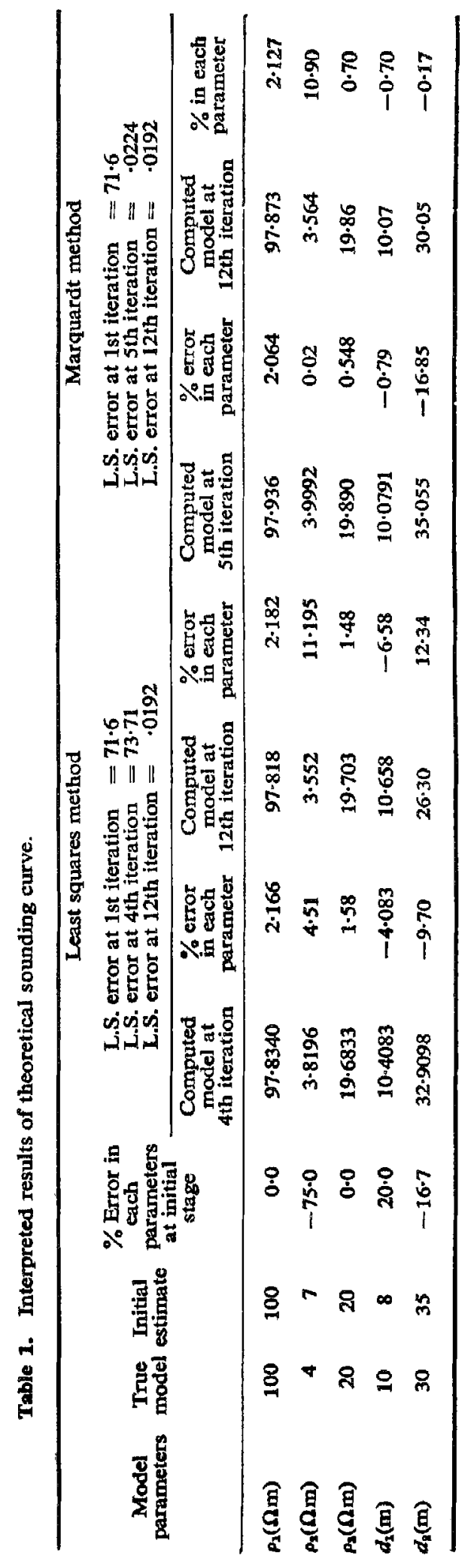




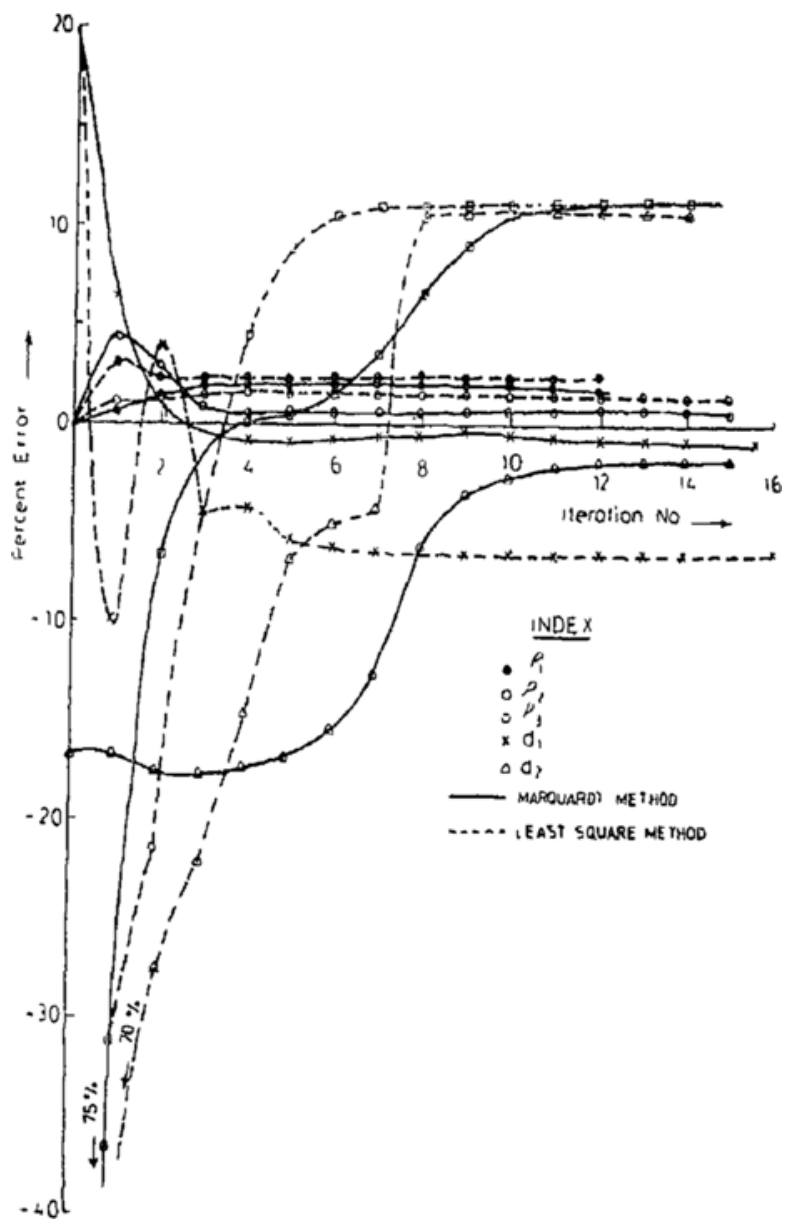

Figure 3. Comparative convergence diagram of parameters $\rho_{1}, \rho_{2}, \rho_{s}, d_{1}$ and $d_{1}$ for curve in figure 1 using least squares method and Marquardt's method.

curves considered is located near villages Dewari $\left(25^{\circ} 28^{\prime} 54^{\prime \prime} \mathrm{N} ; 81^{\circ} 10^{\prime} 25^{\prime \prime} \mathrm{E}\right)$ and Bhadedu $\left(25^{\circ} 26^{\prime} 19^{\prime \prime} \mathrm{N} ; 81^{\circ} 8^{\prime} 24^{\prime \prime} \mathrm{E}\right)$. Geologically the area is occupied by the sands/clays of Gangetic alluvium, beneath which Bundelkhand granites are expected. The nearest exposure of granites are found at Pahari $\left(25^{\circ} 20^{\prime} \mathrm{N} 80^{\circ} 58^{\prime} \mathrm{E}\right)$ the Block headquarters and Chakaundh $\left(25^{\circ} 16^{\prime} \mathrm{N}, 80^{\circ} 56^{\prime} 30^{\prime \prime} \mathrm{E}\right)$ village to the south of Pahari. The alluvium mainly consists of clay and silts mixed with Kankar and varies considerably in thickness. Bands of sands, fine to coarse grained, are also found to be present.

On the basis of visual analysis of the two resistivity curves (figures 4,5 ) the starting models are chosen for use in Marquardt's method. Table 2 represents the initial estimation and interpreted model parameters for the considered two field curves. In figures 4 and 5 the starting model, the final model obtained and the computed resistivity transforms in each case are displayed. The figures show that in both the cases Marquardt's method yields models whose resistivity transform curves coincide well with the observed resisitivity transform curve.

The sounding curve near village Dewari (figure 4) is interpreted using two diffe- 


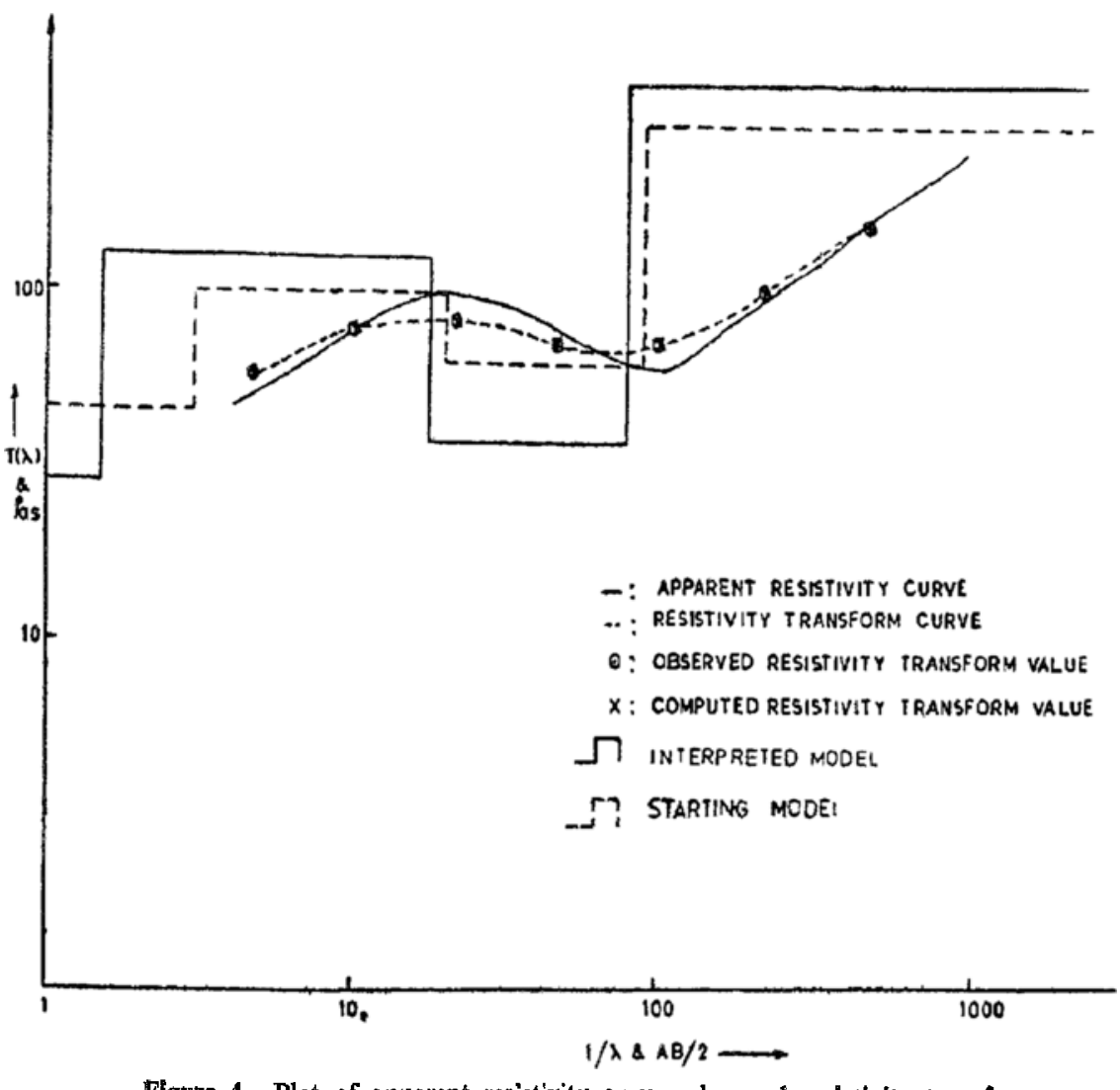

Figure 4. Plot of apparent resistivity curve, observed resistivity transform curve, starting model and interpreted model for sounding near village Dewari, district Banda.

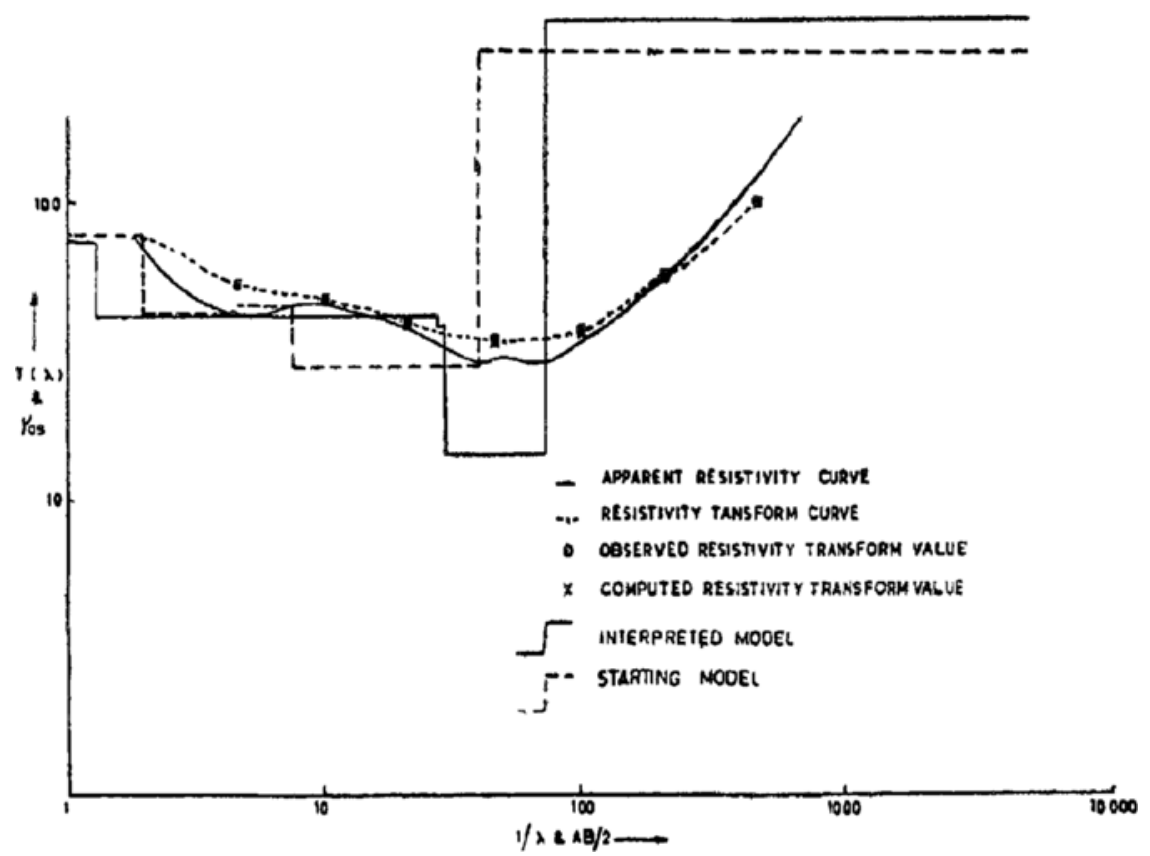

Figure 5. Plot of apparent resistivity curve, observed resistivity, transform curve, starting model and interpreted model for sounding near village Bhadedu, district Banda. 


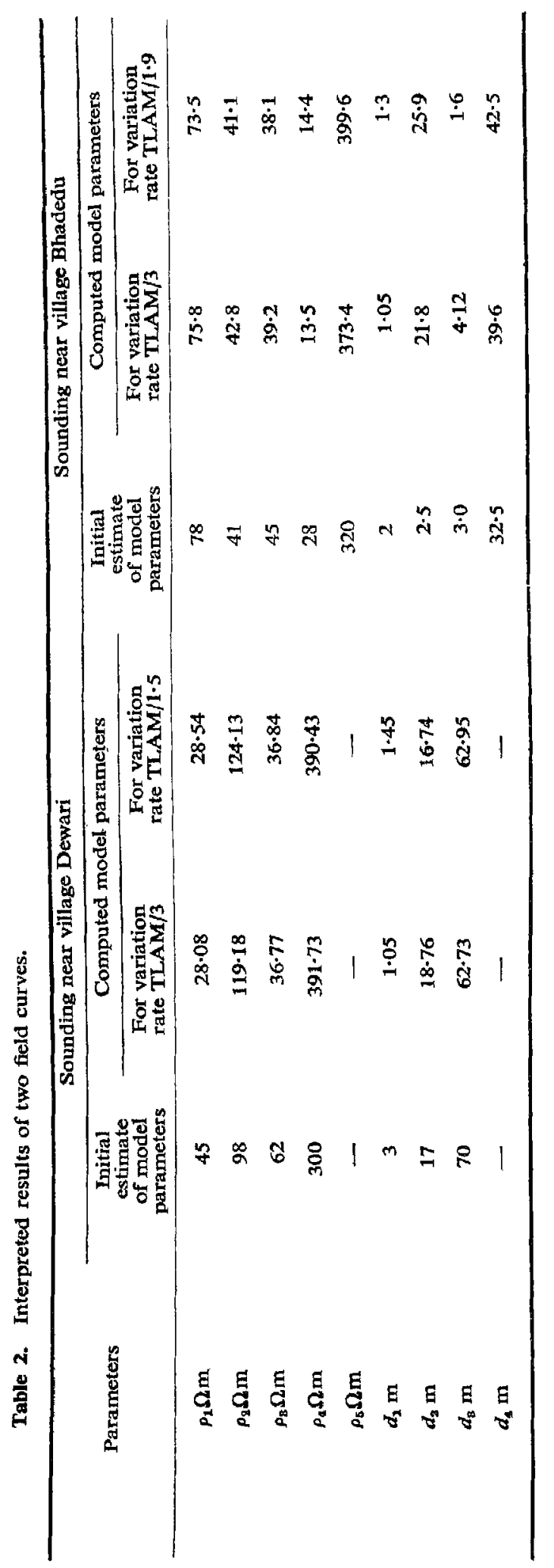


rent rates of variation of TLAM values in each advancing iteration, TLAM 1.5 and TLAM/3. The convergence is obtained in 12 iterations $\left(T_{D}=5.02\right)$ and in 33 iterations $\left(T_{D}=0.0663\right)$ in the case of variation rate TLAM/3 and TLAM/1.5 respectively which suggests that for more precision the variation rate of TLAM/1.5 may be chosen. The same variation rates are used for sounding curve near Bhadedu (figure 5) and the inverse solution is obtained in 17 iterations $\left(T_{D}=6.5\right)$. The interpreted data for these two cases are tabulated in table 2 .

\section{Conclusion}

On the basis of the above discussion we can conclude that the solution of the inverse resistivity problem using resistivity transform function as an intermediary step and based on the linearization of (2) can be most effectively obtained using Marquardt's method in comparison to steepest descend and least squares method. Marquardt's method enables the region of convergence for the initial estimation to be expanded in comparison to least squares method and consequently the scope of the application of the linearization methods is extended to the problem of investigation of geoelectric structures. The essential advantage of using resistivity transform function as an intermediary step lies in the fact that it does not depend on the electrode configuration used, as it is the function of only layer parameters. Thus the present scheme can be effectively employed for any electrode system with minor modification in program pertaining to the extraction of resistivity-transform function from apparent resistivity curve. However, the investigation pertaining to the limitation of the method in case of very thin layer problem will be necessary and this is being further studied by us.

\section{Acknowledgement}

The authors are extremely thankful to Dr V K Gaur and Dr K N Khattri for their constant encouragement and to Sri D C Singhal for providing field data and constructive discussions. They are also thankful to Sri P K Gupta for his help at the computational stage.

\section{References}

Bichara M and Lakshmanan I 1976 Geophys. Prospect. 24354

Ghosh D P 1971 Geophys. Prospect. 19192

Inman J R, Ryu J and Ward S H 1973 Geophysics 381088

Johanson H K 1977 Geophys. Prospect. 25667

Koefoed 01970 Geophys. Prospect. 18564.

Koefoed O 1979 Geosounding principles 1 (Amsterdam: Elsevier)

Marquardt D W 1963 J. Soc. Ind. Appl. Math. 11431

Pek J and Červ V 1979 Studia Geopyhs. Geod. 23349

Slichter L B 1933 Physics 4307

Zohdy A A R 1974 Geol. Surv. Bull. 1313-E 71 